IJ Mazela, 'T Szczapa, 'K Chmura, ${ }^{2} \mathrm{M}$ Kulza, ${ }^{2} \mathrm{E}$ Florek, ${ }^{3} \mathrm{~T}$ Sosnowski, ${ }^{3} \mathrm{~A}$ Moskal, IJ Gadzinowski. 'Department of Neonatology; ' Environmental Toxicology Lab, Poznan University of Medical Sciences, Poznan; ${ }^{3}$ Warsaw University of Technology, Warsaw, Poland

Background and Aims Helium-oxygen gas mixture (heliox) has been utilized for ventilatory support of infants with respiratory failure. Therapies which potentially could improve aerosol delivery to ventilated infants are needed. The aim of the study was to assess heliox utilization in delivery of aerosolized albuterol sulfate to a model of ventilated newborn.

Methods A neonatal system was assembled utilizing an Avea ventilator, test lung and vibrating mesh nebulizer Aeroneb placed within inspiratory arm of the ventilator circuit. Pressure controlled mode(SIMV) was used with a rate of $40 \mathrm{bpm}$, IT of $0.35 \mathrm{~s}$ and inspiratory pressures of $20 \mathrm{cmH}_{2} \mathrm{O}$ with two different ET tubes:3.0 and 2.0.The study was conducted with heliox and air-oxygen gas mixtures at $\mathrm{FiO}_{2}=0.21$. Albuterol sulfate $(0.25 \mathrm{mg} / \mathrm{mL})$ was aerosolized and captured on HME low volume filter located at the end of the ET tube and upstream from the test lung. Emitted dose of the nebulizer was determined by exposing 3 filters to the aerosolized albuterol at the ' $\mathrm{Y}$ ' and end of the ET tube. Filters were collected, extracted and albuterol quantified using high performance liquid chromatography.

Results No significant difference between heliox and air-oxygen was found in albuterol sulfate delivery at the Y-connector and ET tube 3.0.For smaller tube heliox was inferior to air $(\mathrm{p}<0.05)$.

Conclusions Due to its lower density, heliox flow rate at a given driving pressure will be higher than the air-oxygen flow rate. Increased inspiratory flow rate in a patient ventilated using artificial airway may not be beneficial for aerosol delivery. Differences in flow rates between heliox and air-oxygen in the study might have outweighed the previously reported beneficial effects of heliox on aerosol delivery.

\section{DIFFERENT ENDOTRACHEAL TUBES, DIFFERENT CONNECTORS, DIFFERENT LENGTHS: IMPACT ON RESISTANCE AND DEAD SPACE}

doi:10.1136/archdischild-2012-302724.1792

E Kazanci, F Kulali, N Altuntas, S Unal, S Aktas, E Ergenekon. Pediatrics, Newborn Medicine, Gazi University Hospital, Ankara, Turkey

Background and Aims During ventilation, size and length of endotracheal tube affects the dead space (DS) and resistance which is considered important for preterm infants. We aimed to investigate impact of the tube type, length, connector and size on DS and resistance when all variables are controlled on a manikin.

Methods We ventilated a newborn manikin with Draeger Babylog 8000 using regular and surfactant tubes, regular and closed system aspiration connectors. Pressures, inspiratory time and flow were kept constant. Measurements were made at full length of the tubes and after shortening (11 and $13 \mathrm{~cm}$ for 2.5-3.0 and 3.5-4.0 mm tubes respectively) when tidal volume (TV) and resistance displayed on the ventilator were recorded. Differences in TV represented differences in DS.

Results With the use of closed system aspiration connector resistance was elevated by $12 \%$ in all tubes and DS of all tubes except $3.0 \mathrm{~mm}$ tube were increased by $10 \%$. Shortening the tube reduced DS by $1-10 \%$ in all sizes except $2.5 \mathrm{~mm}$ tube. Resistance was higher in long tubes by $4-27 \%$ however this was less than expected. Resistance of 2.5 and $3.5 \mathrm{~mm}$ regular tubes were $2-48 \%$ higher and $3.0 \mathrm{~mm}$ tube was 1-17.6\% lower than surfactant tubes. Resistance was higher in smaller tubes but the magnitude of difference was not as big as the theoretical assumption about the impact of radius to the $4^{\text {th }}$ power.
Conclusion Many variables impact DS and airway resistance during ventilation some of which maybe important for preterm newborn.

\section{EFFECT OF CHEST COMPRESSIONS ON MASK LEAK DURING NEONATAL RESUSCITATION: A MANIKIN STUDY}

doi:10.1136/archdischild-2012-302724.1793

'B Schwaberger, 1,2GM Schmölzer, 'C Binder, 'W Müller, 'B Urlesberger, ' $G$ Pichler. ${ }^{1}$ Department of Paediatrics, Medical University of Graz, Graz, Austria; '2Department of Pediatrics, University of Alberta, Edmonton, AB, Canada

Background Chest compressions during neonatal resuscitation are an infrequent event during neonatal resuscitation.

Objectives To investigate if chest compressions (CC) during simulated neonatal cardio-pulmonary resuscitation affect percentage of mask leak.

Methods 21 neonatal staff members (11 Neonatal nurses, 10 Neonatologists) delivered positive pressure ventilation (PPV) to a modified, leak-free manikin via face mask using a Neopuff T-piece device. After 90 seconds of PPV-only (baseline) an assistant provided chest compressions (CC) at a ratio of $3: 1$ chest compressions to inflations for further 90 seconds $(P P V+C C)$. Mask leak was measured with a Florian respiratory function monitor. Results between $P P V$-only and $P P V+C C$ were compared using dependent t-test for paired samples. Results A total of 2726 ventilations and 3058 chest compressions were analyzed. Overall the mean (SD) mask leak during $P P V$-only and $P P V+C C$ was similar $35( \pm 30) \%$ versus $40( \pm 33) \%(p=0.2)$. However, in the neonatal nurse group mask leak significantly increased from $27( \pm 26) \%$ in the PPV-only group to $43( \pm 35) \%$ in the $P P V+C C$ group ( $\mathrm{p}=0.04)$. In comparison, mask leak was similar in the neonatologist group in the PPV-only and $P P V+C C$ group $42( \pm 33) \%$ and $37( \pm 34) \%(p=0.65)$, respectively.

Conclusions Overall, mask leak did not change once chest compressions were started. However, mask leak significantly increased in the neonatal nurse group once chest compression were provided, but remained similar in the neonatologist group.

\section{DOES SITE OF PULSE OXIMETER PROBE PLACEMENT AFFECT OBSERVED SATURATION VALUES IN BABIES WITH PDA OR RESPIRATORY SUPPORT?}

doi:10.1136/archdischild-2012-302724.1794

'P Suresh, ${ }^{2}$ W Cheadle, ${ }^{2,3}$ S Gupta. 'Medical School, Newcastle University, Newcastleupon-Tyne; '2Neonatology \& Paediatrics, University Hospital of North Tees; ${ }^{3}$ School of Medicine \& Health, Durhum University, Stockton-on-Tees, UK

Background Pulse oximetry is routinely utilised for monitoring oxygen saturations in newborn babies. The site for pulse oximeter probe placement is randomly selected and saturations targeted according to the unit policy.

Aim To study whether site of pulse oximeter probe placement would affect observed saturation values in preterm babies on respiratory support and or having patent ductus arteriosus (PDA)?

Methods Babies born < 32 weeks gestation and admitted to the tertiary level neonatal unit were randomly included in this prospective pilot study. They were allocated to one of 4 groups (Figure 1). One probe was placed on right upper limb (pre-ductal) and other on lower limb (post-ductal). Simultaneous continuous saturation recording was done for at least 6 hours using Radical-7, Masimo ${ }^{\circledR}$ pulse oximeters that recorded data every 2 seconds. At the end of the recording period data was downloaded using the software and analysed using SPSS $®$ version 19.

Results Twenty babies were enrolled in this study and $\sim 500,000$ data points recorded. 
The mean pre-ductal saturations were lower than post-ductal saturations in all groups except the group not having a PDA and not on respiratory support (figure 1).

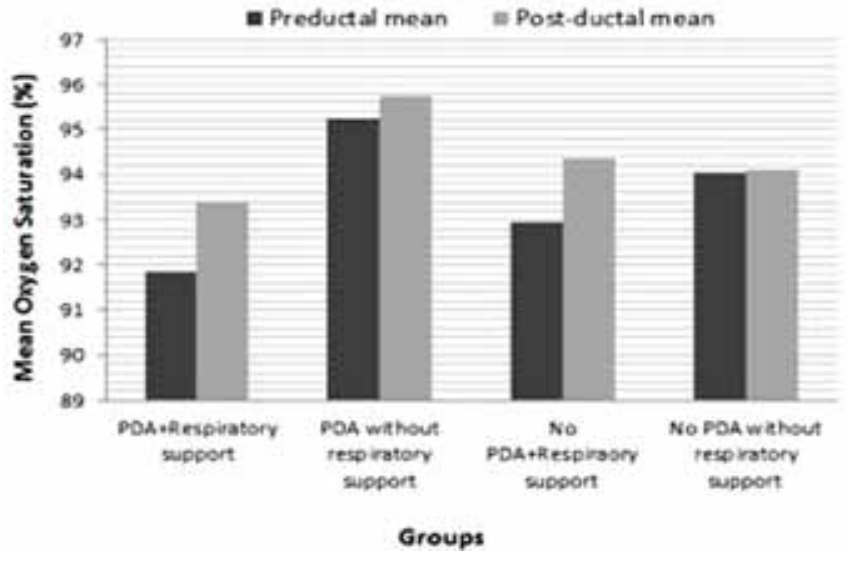

Abstract 1794 Figure 1

Conclusions The site of measurement can affect the observed oxygen saturation values and hence should be taken in account while bedside monitoring and planning clinical trials.

\section{DID VENTILATORY STRATEGY CHANGE DURING THE LAST 5 YEARS IN ITALIAN NEONATAL NETWORK?}

doi:10.1136/archdischild-2012-302724.1795

${ }^{1} \mathrm{~V}$ Vendettuoli, ${ }^{2} \mathrm{M}$ Condò, ${ }^{3} \mathrm{~A}$ Poloniato, ${ }^{4} \mathrm{M}$ Raia, ${ }^{5} \mathrm{~F}$ Ramacciato, ${ }^{6} \mathrm{LG}$ Tina, ${ }^{7} \mathrm{LM}$ Abbiati, ${ }^{8} \mathrm{~A}$ Staffler, ${ }^{9} \mathrm{~S}$ Agostiniani. 'NICU Fondazione IRCCS Ca' Granda Ospedale Maggiore Policlinico - Università degli Studi di Milano, Milan; ${ }^{2}$ Division of Neonatology and NICU, Ospedale A. Manzoni, Lecco; ${ }^{3}$ Unità Operativa di Neonatologia e Patologia Neonatale Dipartimento Materno-Infantile - H San Raffaele, Milan; ${ }^{4}$ SCDU Neonatologia ASO OIRM S. Anna Università di Torino, Torino; ${ }^{5}$ Division of Neonatology and NICU Ospedale Cardarelli, Campobasso; ${ }^{6}$ TIN ARNAS Ospedale Garibaldi, Catania; ${ }^{7}$ U.O. Neonatologia e Terapia Intensiva Neonatale - Fondazione MBBM - Ospedale San Gerardo Monza, Monza; ${ }^{8}$ Department of Neonatology, Regional Hospital of Bolzano, Bolzano; ${ }^{9} \mathrm{AOU}$ MEYER, Firenze, Italy

Background Intubation and mechanical ventilation (MV) are lifesaving procedures but are associated with a higher incidence of acute and chronic complications. Thus, non-invasive ventilation (NIV: nasal continuous pulmonary distending pressure, nasal ventilation, or high-flow nasal cannula) is increasingly used.

Aim To evaluate changes in ventilatory strategies between 2006 and 2010 in Italian neonatal network (INN).

Methods A cohort of neonates $<30$ weeks gestational age (GA) or $<1501 \mathrm{~g}$ birth weight (BW), without congenital anomalies, born in 2006 and 2010, assisted in 31 hospitals participating in INN both years, was analysed ( $N=3459$ : 1713 in 2006, and 1746 in 2010). Variables were defined according to Vermont-Oxford network. Logistic regressions, adjusting for confounders (GA, BW for GA, antenatal steroids, mode of delivery, multiple pregnancy, 1-minute Apgar score, being inborn, sex, intubation in delivery room, RDS, PDA), and clustering for hospitals, were used.

Results Between 2006 and 2010 there were no changes in GA or BW (2006: mean GA 29.1 wks; BW 1087 g; 2010: GA 29.2 wks; BW $1083 \mathrm{~g}$ ), while antenatal steroids increased (from $78.5 \%$ to $83.5 \%$ ). The number of infants receiving any ventilatory support increased from $81.8 \%$ to $85.9 \%$. After adjusting for confounders, mortality decreased (Odds ratio $=0.75,95 \%$ confidence interval $0.57-0.98$ ) as well as mechanical ventilation $(\mathrm{OR}=0.72,95 \% \mathrm{CI} 0.57-0.90)$ and $\mathrm{BPD}(\mathrm{OR}=0.68,95 \% \mathrm{CI}$ 0.54-0.86), while NIV increased $(\mathrm{OR}=1.70$, $95 \%$ CI 1.41-2.04).
Conclusions In the last 5 years, we observed a reduction of MV and an increase of NIV use. This was accompanied by a decrease in risk-adjusted mortality and $\mathrm{BPD}$.

\section{INCIDENCE OF AND RISK FACTORS FOR AIR LEAKS IN PRETERM INFANTS EXPOSED TO RESTRICTIVE USE OF ENDOTRACHEAL INTUBATION}

doi:10.1136/archdischild-2012-302724.1796

${ }^{1} \mathrm{H}$ Hummler, ${ }^{1} \mathrm{E}$ Parys, ${ }^{1} \mathrm{~J}$ Essers, ${ }^{1} \mathrm{R}$ Hopfner, ${ }^{1} \mathrm{O}$ Beringer, ${ }^{2} \mathrm{~B}$ Mayer, ${ }^{1} \mathrm{H}$ Fuchs, ${ }^{1} \mathrm{M}$ Schmid. ${ }^{1}$ Dept. of Pediatrics, Children's Hospital, University of Ulm; ${ }^{2}$ Institute of Epidemiology and Medical Biometry, University of UIm, UIm, Germany

Introduction The occurrence of air leaks such as pneumothorax (PTX), pneumopericardium (PPC) and pulmonary interstitial emphysema (PIE) may be a life-threatening condition in preterm infants.

Aim of the Study To study the incidence of and risk factors for air leaks in preterm infants treated with a policy of sustained inflations followed by non-invasive ventilation in the delivery room.

Methods Perinatal variables, variables of delivery room support and respiratory support in the NICU were analyzed retrospectively for infants with/without air leaks in preterm infants $<30$ wks GA born 2005-2009 ( $n=297$ )

Results Median (range) gestational age was $26+0(22+4-29+1)$ wks, birth weight was 790 (265-1660) g and 270/297 (91.0\%) survived. 63 (21.2\%) developed any air leak, 32 (10.8\%) developed PTX, $44(14.8 \%)$ PIE, and $1(0.3 \%)$ PPC. Infants with air leaks had a higher risk for death $(18(28.6 \%)$ vs. 9 (3.8\%), p<0.01) and for IVH Grade 3-4 (16 (25.4\%) vs. 29 (12.4\%), p<0.05). Air Leaks were associated with less use of prenatal steroids (44 (69.8\%) vs. 199 (85.4\%), $\mathrm{p}<0.01)$ and a more common use of cardiac compressions $(9(14.3 \%)$ vs. $11(4.7 \%), \mathrm{p}<0.01)$, use of a pressure of $30 \mathrm{cmH}_{2} \mathrm{O}$ for sustained inflations (32 (55.2)\% vs. $80(36.7 \%), \mathrm{p}<0.05)$ and intubation during initial resuscitation (34 (54.0\%) vs. 60 (25.6\%), p<0.01)

Conclusion Air leaks were associated with an increased risk for mortality and severe IVH. Our approach resulted in a high rate of survival but was associated with a substantial rate of air leaks. Randomized trials are necessary further improve delivery room care.

\section{DO NON-INVASIVE VENTILATORY STRATEGIES WORK IN MICRO-PREMATURE INFANTS WHO ARE AT THE LIMITS OF VIABILITY?}

doi:10.1136/archdischild-2012-302724.1797

E Okulu, S Arsan, IM Akin, S Alan, A Kılıç, B Atasay. Department of Pediatrics, Division of Neonatology, Ankara University, Ankara, Turkey

Aim To evaluate the non-invasive ventilatory support in micropremature infants who are at the limits of viability.

Methods This prospective cohort study from January-2009 to December 2011 included infants born before 26 weeks'. During resuscitation, stabilisation and transport infants were ventilated with a T-piece resuscitator, and all received prophylactic surfactant at a dose of $100 \mathrm{mg} / \mathrm{kg}$. If respiratory drive was present, infants were extubated to NCPAP. The demographic and clinical features of the infants were assessed.

Results Twenty-four infants born during the study period. Antenatal steroid rate was $16.7 \%$. Mean gestational age(GA) and birth weight(BW) were $24.3 \pm 0.9$ weeks, and $660.2 \pm 125.5 \mathrm{~g}$, respectively. The presence of premature rupture of membranes and chorioamnionitis rate was $54 \%$. Only five $(21 \%)$ of 24 infants could be extubated to NCPAP, and three of these five were intubated in first 3-days. Only two(8.3\%) infants succeeded on NCPAP, and the GAs' were 24.6 and 25.1 weeks, the BWs' were 1010 and $730 \mathrm{~g}$. The rate of NEC, PDA, İVH and pulmonary hemorrhage were 29\%, 36\%, 36\% and $21 \%$, respectively in infants who survived more than 2 days. 